\title{
Prognosis of patients with Acinetobacter baumannii infection in the intensive care unit: A retrospective analysis
}

\author{
DONG XIAO $^{1}$, LU WANG $^{1}$, DAQUAN ZHANG $^{1}$, DONGMING XIANG $^{1}$, QI LIU $^{1}$ and XUEZHONG XING ${ }^{2}$ \\ ${ }^{1}$ Second Department of Critical Care Medicine, People's Hospital of Xinjiang Uygur Autonomous Region, \\ Urumqi, Xinjiang 830001; ${ }^{2}$ Department of Critical Care Medicine, Cancer Hospital, \\ Chinese Academy of Medical Sciences \& Peking Union Medical College, Beijing 100021, P.R. China
}

Received December 15, 2014; Accepted January 15, 2016

DOI: $10.3892 / \mathrm{etm} .2017 .4137$

\begin{abstract}
The present retrospective study aimed to investigate the prognostic factors for critically ill patients with an Acinetobacter baumannii (A. baumannii) infection. A total of 37 patients from the intensive care unit (ICU) were enrolled in the study. Data was collected from patients, including age, gender, ethnicity, A. baumannii infection status, concurrent infection status, prescribed antibiotics, Acute Physiology and Chronic Health Evaluation II (APACHE II) and sepsis-related organ failure assessment (SOFA) scores, procalcitonin level, site of infection, shock, sepsis and renal replacement therapy status. Univariate and multivariate analyses were performed to determine the prognostic factors for mortality. In total, 8 out of the 37 patients included in this trial did not survive, resulting in an ICU mortality rate of $21.6 \%$. Univariate analysis indicated that, in comparison with the survival group, APACHE II and SOFA scores were significantly higher in the mortality group $(\mathrm{P}<0.002$ and $\mathrm{P}<0.001$, respectively). A larger number of patients with septic shock were detected in the mortality group in comparison with the survival group ( $n=4$ and $n=3$, respectively), whereas a larger number of patients were infected with multidrug-resistant A. baumannii (MDRAB) in the survival group in comparison with the mortality group (51.9 and $14.3 \%$, respectively). However, the prognosis was not significantly influenced by antibiotics administered, mixed infection or the site of infection. Multivariate analysis demonstrated that the APACHE II score was the only independent factor affecting the disease prognosis. Receiver operating characteristic curve analysis of the APACHE II score demonstrated that the area under the curve was $0.845 \pm 0.078$. In patients with an APACHE II score
\end{abstract}

Correspondence to: Dr Dong Xiao, Second Department of Critical Care Medicine, People's Hospital of Xinjiang Uygur Autonomous Region, 91 Tianchi Road, Urumqi, Xinjiang 830001, P.R. China E-mail: xiaodongzhurensicu@163.com

Key words: prognosis, retrospective analysis, critically ill patients, Acinetobacter baumannii of 15 , the sensitivity and specificity predictions for mortality were $87.5 \%$ and $72.4 \%$, respectively. The APACHE II score at diagnosis was the only independent factor found to assist in the prognosis of mortality for patients in the ICU with an A. baumannii infection. In conclusion, the results of the present study demonstrated that the APACHE II score may contribute towards the prognostic evaluation of patients in the ICU with an A. baumannii infection.

\section{Introduction}

The effects of hospital-acquired infections on the outcome of critically ill patients have been extensively investigated. Acinetobacter baumannii (A. baumannii), a Gram-negative aerobic coccobacillus, has emerged as one of the leading causes for nosocomial bloodstream infections in intensive care units (ICUs) (1). A. baumannii has been demonstrated to cause a number of clinical infections, including bacteremia, pneumonia, meningitis and surgical site infections $(2,3)$.

A. baumannii has been involved in infections with increased mortality rates (4). The virulence of $A$. baumannii can be enhanced by the occurrence of multiple antimicrobial resistance, resulting in difficulties when determining the therapeutic options to treat the infection $(5,6)$. It has been reported that multidrug-resistant $A$. baumannii induces a fulminant infection following the treatment of a surgical wound (7). According to the Chinese Meropenem Susceptibility Surveillance (CMSS) report in 2010 (8), a total of 180 strains of A. baumannii have been identified in 1259 isolates of Gram-negative bacilli from 13 hospitals; this makes the infection second only to Pseudomonas aeruginosa (8). In addition, the susceptibility of A. baumannii to carbapenems is $<37.0 \%$ and its susceptibility to minocycline is $47.8 \%$, while the incidence of extensively drug-resistant A. baumannii is $60.1 \%$ (8). Furthermore, the mortality rates of patients with A. baumannii infection have been reported to be $26.0-55.7 \%$, and the attributable mortality rates were $8.4-36.5 \%$ (9).

Currently, there are few studies concerning the impact of A. baumannii infection on the prognosis of critically ill patients in China $(10,11)$. In the present retrospective study, the characteristics of patients with A. baumannii infections in the ICU are investigated, and the clinical prognostic factors for A. baumannii infection are analyzed. 


\section{Materials and methods}

Study population. The present retrospective study enrolled patients with an A. baumannii infection who were admitted to the ICU of the People's Hospital of Xinjiang Uygur Autonomous Region (Urumqi, China) between January 2013 and December 2013 for the treatment of infection. According to the Expert Consensus Document on A. baumannii infection diagnosis, treatment and prevention in China (12), multidrug-resistant A. baumannii (MDRAB) were defined as the strains that were resistant to at least three classes of the following antimicrobial agents: Cephalosporins, carbapenems, sulbactam, fluoroquinolones and aminoglycoside drugs. Mixed infection occurred when other bacteria and/or fungi were detected in the culture in addition to A. baumannii. Written informed consent was obtained from patients, and the study was approved by the ethics committee of the People's Hospital of Xinjiang Uygur Autonomous Region.

Data collection. The following data were obtained for each patient: Age, gender, ethnicity, A. baumannii infection status, concurrent infection status, antibiotics for therapeutic application, severity of the disease, serum procalcitonin (PCT) level, the site of infection, shock, sepsis and renal replacement therapy status. The severity of the disease was evaluated based on the Acute Physiology and Chronic Health Evaluation II (APACHE II) and sepsis-related organ failure assessment (SOFA) scores.

The APACHE II scoring system covered 12 routine physiologic measurements, including age and previous health status, that provided a general evaluation of the disease severity (range, 0-71). A high score indicated poor prognosis, and this could be used to predict the mortality of patients (13). The SOFA score (range, 1-4) was used to assess the course of organ dysfunction/failure in critically ill patients, including the respiratory system, coagulation system, liver, cardiovascular system, central nervous system and kidney. A SOFA score of $\leq 2$ indicated organ dysfunction, and a SOFA score of $\geq 3$ indicated organ failure. High SOFA scores for individual organs were associated with increased mortality (14). Shock was defined as mean blood pressure $<65 \mathrm{mmHg}$ in spite of an adequate quantity of fluids ( $\leq 1000 \mathrm{ml}$ crystalloids or $500 \mathrm{ml}$ colloids) (15). Sepsis, severe sepsis and septic shock were diagnosed according to the guidelines of the $2001 \mathrm{SCCM} / \mathrm{ESICM} / \mathrm{ACCP} / \mathrm{ATS} / \mathrm{SIS}$ International Sepsis Definitions Conference (16).

Statistical analysis. Data are expressed as the mean \pm standard deviation or n (\%). SPSS version 16.0 software (SPSS, Inc., Chicago, IL, USA) was used to perform statistical analysis. Intergroup comparisons were performed using the t-test or $\chi^{2}$-test. Logistic regression analysis was used to determine the factors affecting the ICU mortality. Receiver operating characteristic (ROC) curve analysis was performed to determine the cut-off point of the APACHE II score. $\mathrm{P}<0.05$ was considered to indicate a statistically significant difference.

\section{Results}

Univariate analysis. A total of 37 patients in the ICU (male, 28; female, 9; age range, 34-70 years) were included in the present
Table I. Acinetobacter baumannii culture sources.

Culture source

Cases, n (\%)

\begin{tabular}{lc}
\hline Sputum & $34(91.9)$ \\
Peritoneal drainage fluid & $1(2.7)$ \\
Urine & $1(2.7)$ \\
Wound secretion & $1(2.7)$ \\
\hline
\end{tabular}

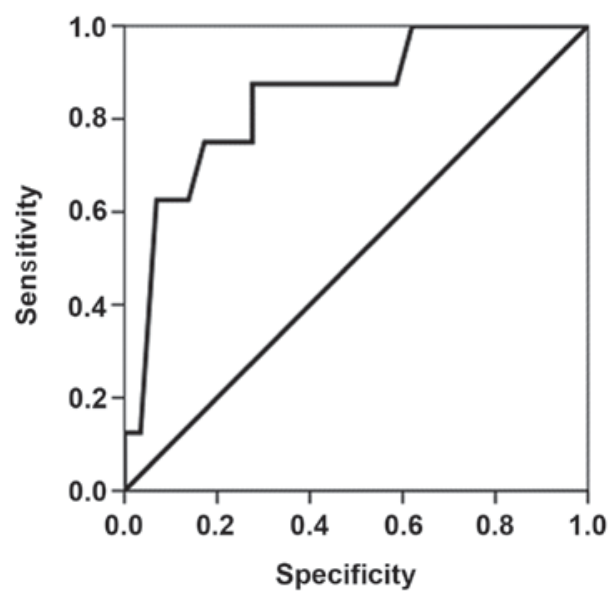

Figure 1. Receiver operating characteristic (ROC) curve analysis. ROC curve was constructed for the acute physiology and chronic health evaluation II score in the prognosis of ICU patients with A. baumannii infection, and the area under the curve was calculated accordingly.

study. The sample culture was primarily derived from the sputum, peritoneal drainage fluid, urine and wound secretion (Table I). Of the 37 patients, 8 patients did not survive, resulting in an ICU mortality rate of $21.6 \%$.

As presented in Table II, univariate analysis indicated that, in comparison with the survival group, the APACHE II and SOFA scores were significantly higher in the mortality group $(\mathrm{P}<0.002$ and $\mathrm{P}<0.001$, respectively). In addition, a great number of patients with septic shock were observed in the mortality group in comparison with the survival group $(n=4$ and $n=3$, respectively), and a greater number of patients were infected with MDRAB in the survival group compared with those in the mortality group (51.9 and $41.3 \%$, respectively). However, there were no statistically significant differences in the age, gender, ethnicity or PCT level between the two groups. Furthermore, the prognosis was not significantly influenced by the various antibiotics used for therapeutic applications, the presence of mixed infection or the site of infection.

Multivariate analysis. The results of multivariate analysis demonstrated that the APACHE II score of the patients at diagnosis is the only independent factor that can indicate the disease prognosis (Table III). The SOFA score, septic shock and hospitalization duration were related with the disease prognosis; however, they were not independent prognostic factors. ROC curve analysis was performed in order to determine the cut-off point for the APACHE II score, and the area under the ROC curve (Fig. 1) was found to be $0.845 \pm 0.078$ (95\% confidence interval, 0.692-0.998). In patients with an 
Table II. Univariate analysis of intensive care unit mortality in patients with A. baumannii infection.

\begin{tabular}{|c|c|c|c|c|}
\hline Clinical variable & Survival group $(n=29)$ & Mortality group $(\mathrm{n}=8)$ & $\mathrm{t}$-test $/ \chi^{2}$ & P-value \\
\hline Age, years & $50.17 \pm 19.25$ & $60.62 \pm 15.16$ & -1.414 & 0.166 \\
\hline Males, n (\%) & $23(79.3 \%)$ & $5(62.5 \%)$ & 0.963 & 0.327 \\
\hline Females, n (\%) & $6(20.7 \%)$ & $3(37.5 \%)$ & & \\
\hline Multidrug-resistant A. baumannii, $n(\%)$ & $14 / 27(51.9 \%)$ & $1 / 7(14.3 \%)$ & 3.182 & 0.074 \\
\hline \multicolumn{5}{|l|}{ Antibiotics, n (\%) } \\
\hline Sulbactam & $13(44.8 \%)$ & $3(37.5 \%)$ & 0.137 & 0.711 \\
\hline Cephalosporins & $10(34.5 \%)$ & $4(50.0 \%)$ & 0.642 & 0.423 \\
\hline Carbapenems & $8(27.6 \%)$ & $2(25.0 \%)$ & 0.021 & 0.884 \\
\hline Minocycline & $7(24.1 \%)$ & $2(25.0 \%)$ & 0.003 & 0.960 \\
\hline Mixed infection, n (\%) & & & 3.117 & 0.210 \\
\hline None & $9(31.0 \%)$ & $3(37.5 \%)$ & & \\
\hline Same site & $16(55.2 \%)$ & $2(25.0 \%)$ & & \\
\hline Different sites & $4(13.8 \%)$ & $3(37.5 \%)$ & & \\
\hline APACHE II scores & $11.31 \pm 5.46$ & $18.25 \pm 4.23$ & -3.319 & 0.002 \\
\hline SOFA scores & $5.14 \pm 2.30$ & $8.75 \pm 3.37$ & -3.352 & 0.001 \\
\hline Procalcitonin level, pg/l & $6.25 \pm 15.66$ & $25.03 \pm 37.34$ & -1.209 & 0.277 \\
\hline Shock, n $(\%)$ & $6(6.9 \%)$ & $5(62.5 \%)$ & 5.247 & 0.022 \\
\hline Renal replacement therapy, n (\%) & $2(6.9 \%)$ & $1(12.5 \%)$ & 0.264 & 0.607 \\
\hline Disease severity, n (\%) & & & 6.778 & 0.034 \\
\hline Sepsis & $23(79.3)$ & $4(50.0)$ & & \\
\hline Severe Sepsis & $3(10.3)$ & $0(0.0)$ & & \\
\hline Septic shock & $3(10.3)$ & $4(50.0)$ & & \\
\hline Infection sites, $\mathrm{n}(\%)$ & & & 0.264 & 0.607 \\
\hline Lung & $27(93.1 \%)$ & $7(87.5 \%)$ & & \\
\hline Others & $2(6.9 \%)$ & $1(12.5 \%)$ & & \\
\hline Length of intensive case unit stay, days & $25.90 \pm 22.97$ & $16.12 \pm 6.40$ & 1.180 & 0.246 \\
\hline Length of hospital stay, days & $40.45 \pm 23.18$ & $20.88 \pm 8.63$ & 3.711 & 0.001 \\
\hline
\end{tabular}

A. baumannii, Acinetobacter baumannii; APACHE II, acute physiology and chronic health evaluation; SOFA, sepsis-related organ failure assessment.

Table III. Multivariate analysis of intensive care unit mortality in patients with A. baumannii infection.

\begin{tabular}{lccccc}
\hline Clinical variable & Regression coefficient & Standard error & Wald value & P-value & Relative risk (95\% CI) \\
\hline APACHE II score & 0.275 & 0.116 & 5.570 & 0.018 & $1.316(1.048-1.654)$ \\
Constant & -6.146 & 2.101 & 8.560 & 0.003 & N/A \\
\hline
\end{tabular}

The constant was set to avoid model mis-specification; the SOFA score, septic shock and hospitalization duration were not independent prognostic factors. A. baumannii, Acinetobacter baumannii; APACHE II, acute physiology and chronic health evaluation; CI, confidence interval; N/A, not available.

APACHE II score of 15 , the sensitivity and specificity for the prediction of mortality were 87.5 and $72.4 \%$, respectively.

\section{Discussion}

In a previous study, A. baumannii infections were found to account for $33.94 \%$ of all Gram-negative bacilli infections (17). The resistant rate of A. baumannii to sulbactam is $30-60 \%$, and its resistance rate to carbapenems is $60-80 \%$ (17). In the present study, it was observed that the incidence of MDRAB was $44.1 \%(15 / 34)$, which is in accordance with the results of previous studies. In addition, a greater number of patients infected with MDRAB were detected in the survival group. This may be due to A. baumannii not being the dominant species in certain microbial cultures that were recognized as MDRAB, and thus the infection would not result in mortality. 
A study by Qiao et al (18) also indicated that the percentage of MDRAB was significantly higher in the survival group in comparison with that in the mortality group $(\mathrm{P}<0.05)$.

It has been observed that the genotype of $A$. baumannii contributes towards its susceptibility to antibiotics. ST75 and ST138 A. baumannii containing OXA-23-like genes were shown to be resistant to carbapenem drugs (19). However, A. baumannii with drug-resistant and non-resistant genotypes could not be distinguished by routine laboratory tests currently performed in clinical practice (12). This may explain why a number of researchers reported weak virulence of $A$. baumannii in the clinic, and did not detct any association between A. baumannii infection and disease prognosis $(11,20)$.

A. baumannii is an opportunistic pathogen that is prone to be located at sites such as the skin, conjunctiva, and oral, respiratory, rectal and genitourinary tracts $(12,21,22)$. When a specimen culture detects only A. baumannii, the prediction of whether an infection will occur may be determined by evaluating a number of clinical manifestations, auxiliary examinations and laboratory examinations. However, if other bacteria and/or fungi are detected in the same culture or at other infection sites along with A. baumannii, further clinical tests are required in order to confirm the diagnosis. In the present study, only $33.3 \%$ (12/36) of patients were infected by A. baumannii alone, while $66.7 \%$ (24/36) of patients presented mixed infection at the same or different sites. Comprehensive judgment regarding the type of antibiotics prescribed to patients should, therefore, be applied when treating A. baumannii and other pathogenic microorganisms. The results from the present study demonstrated that the APACHE II score at diagnosis was the only independent prognostic factor in patients with A. baumannii infection, and this is in accordance with the results of Qiao et al (18). However, the present study is single-centered with a limited number of subjects. Further in-depth multi-center studies with larger sample sizes are required in order to elucidate the prognostic factors involved in an A. baumannii infection.

In conclusion, the results of the present study demonstrated that the APACHE II score at diagnosis is an independent factor for the disease prognosis of patients in the ICU with an A. baumannii infection. This prognosis is not significantly influenced by the antibiotics administered for therapeutic applications, mixed infections, MDRAB or the site of infection in these patients. The findings of the present study contribute towards the prognostic evaluation of patients in ICUs with an A. baumannii infection.

\section{Acknowledgements}

The present study was supported by funding from the Key Laboratory of Xinjiang Uygur Autonomous Region (grant no. xjys0906-2014-03).

\section{References}

1. García-Garmendia JL, Ortiz-Leyba C, Garnacho-Montero J, Jiménez-Jiménez FJ, Pérez-Paredes C, Barrero-Almodóvar AE and Gili-Miner M: Risk factors for Acinetobacter baumannii nosocomial bacteremia in critically ill patients: A cohort study. Clin Infect Dis 33: 939-946, 2001.

2. Jaggi N, Sissodia P and Sharma L: Control of multidrug resistant bacteria in a tertiary care hospital in India. Antimicrob Resist Infect Control 1: 23, 2012.
3. Falagas ME and Rafailidis PI: Attributable mortality of Acinetobacter baumannii: No longer a controversial issue. Crit Care 11: 134, 2007.

4. Lemos EV, de la Hoz FP, Einarson TR, McGhan WF, Quevedo E, Castañeda $\mathrm{C}$ and Kawai $\mathrm{K}$ : Carbapenem resistance and mortality in patients with Acinetobacter baumannii infection: Systematic review and meta-analysis. Clin Microbiol Infect 20: 416-423, 2014.

5. Grupper M, Sprecher H, Mashiach $T$ and Finkelstein R: Attributable mortality of nosocomial Acinetobacter bacteremia. Infect Control Hosp Epidemiol 28: 293-298, 2007.

6. Aydemir H, Celebi G, Piskin N, Oztoprak N, Keskin AS, Aktas E, Sumbuloglu V and Akduman D: Mortality attributable to carbapenem-resistant nosocomial Acinetobacter baumannii infections in a Turkish university hospital. Jpn J Infect Dis 65: 66-71, 2012

7. Maragakis LL, Cosgrove SE, Song X, Kim D, Rosenbaum P, Ciesla N, Srinivasan A, Ross T, Carroll K and Perl TM: An outbreak of multidrug-resistant Acinetobacter baumannii associated with pulsatile lavage wound treatment. JAMA 292: 3006-3011, 2004.

8. Wang H, Zhao CJ, Wang ZW, Ni YX, Chen MJ, Xu YC, Yu YS, Zhang LY, Mei YN, Chu YZ, et al: Report from Chinese Meropenem Susceptibility Surveillance in 2010: Antimicrobial resistance among nosocomial Gram-negative bacilli. Zhong Hua Jian Yan Yi Xue Za Zhi 34: 897-904, 2011 (In Chinese).

9. Falagas ME and Rafailidis PI: Attributable mortality of Acinetobacter baumannii: No longer a controversial issue. Crit Care 11: 134, 2007.

10. Shi Y, Xu YC, Liu Y, Du W, Rui X and Wang Y: Cefoperazone-sulbactam plus minocycline in the treatment of extentively drug resistant Acinetobacter infections. Zhong Hua Yi Xue Za Zhi 92: 2847-2850, 2012 (In Chinese).

11. Yu Z, Zhang D, Yan JJ and Zhou QS: Acinetobacter baumannii infection in intensive care unit: A retrospective analysis. Wu Han Da Xue Xue Bao (Yi Xue Ban) 34: 895-898, 2013 (In Chinese).

12. Chen B, He LX, Hu B, Ni YX, Qiu H, Shi Y, Shi Y, Wang H, Wang M, Yang Y, et al: Expert Consensus Document on Acinetobacter baumannii infection diagnosis, treatment and prevention in China. Zhong Hua Yi Xue Za Zhi 92: 76-85, 2012 (In Chinese).

13. Knaus WA, Draper EA, Wagner DP and Zimmerman JE: APACHE II: A severity of disease classification system. Crit Care Med 13: 818-829, 1985.

14. Vincent JL, Moreno R, Takala J, Willatts S, De Mendonca A, Bruining H, Reinhart CK, Suter PM and Thijs LG: The SOFA (Sepsis-related organ failure assessment) score to describe organ dysfunction/failure. On behalf of the working group on sepsis-related problems of the European society of intensive care medicine. Intensive Care Med 22: 707-710, 1996.

15. Xing XZ, Wang HJ, Huang CL, Yang QH, Qu SN, Zhang H, Wang H, Gao Y, Xiao QL and Sun KL: Prognosis of patients with shock receiving vasopressors. World J Emerg Med 4: 59-62, 2013.

16. Levy MM, Fink MP, Marshall JC, Abraham E, Angus D, Cook D, Cohen J, Opal SM, Vincent JL and Ramsay G; SCCM/ESICM/ACCP/ATS/SIS: 2001 SCCM/ESICM/ACCP/ ATS/SIS International Sepsis Definitions Conference. Crit Care Med 31: 1250-1256, 2003.

17. Wang L, Shi XH and Xiao D: Drug resistance of Acinetobacter baumannii infection in SICU. Xinjiang Medicine 43: 141-142, 2013.

18. Qiao L, Zhang JS, Mei YN, Zhang HZ and Su CL: Analysis of risk factors for the prognosis of Acinetobacter baumannii bloodstream infection. Zhonghua Wei Zhong Bing Ji Jiu Yi Xue 25: 471-474, 2013 (In Chinese).

19. Park YK, Jung SI, Park KH, Kim DH, Choi JY, Kim SH and Ko KS: Changes in antimicrobial susceptibility and major clones of Acinetobacter calcoaceticus-baumannii complex isolates from a single hospital in Korea over 7 years. J Med Microbiol 61: 71-79, 2012.

20. Wang H, Liu D, Chen P, Wang Z and Cheng Y: A survey of risk factors of Acinetobacter baumannii infection in trauma patients and their mortality. Chong Qing Yi Xue 40: 3665-3667, 2011 (In Chinese).

21. Ma MY, Xu J, Yu N and Huang GM: Analysis of drug resistance of Acinetobacter baumannii and its related factors in ICU. Zhonghua Wei Zhong Bing Ji Jiu Yi Xue 25: 686-689, 2013 (In Chinese).

22. Bacakoğlu F, Korkmaz Ekren P, Taşbakan MS, Başarik B, Pullukçu H, Aydemir S, Gürgün A and Başoğlu $O K$ : Multidrug-resistant Acinetobacter baumannii infection in respiratory intensive care unit. Mikrobiyol Bul 43: 575-55, 2009 (In Turkish). 